\title{
Get Your Requirements Straight: Storyboarding Revisited
}

\author{
Mieke Haesen, Kris Luyten, and Karin Coninx \\ Hasselt University - tUL - IBBT, \\ Expertise Centre for Digital Media, \\ Wetenschapspark 2, B-3590 Diepenbeek, Belgium \\ \{mieke.haesen, kris. luyten, karin. coninx\} @uhasselt. be
}

\begin{abstract}
Current user-centred software engineering (UCSE) approaches provide many techniques to combine know-how available in multidisciplinary teams. Although the involvement of various disciplines is beneficial for the user experience of the future application, the transition from a user needs analysis to a structured interaction analysis and UI design is not always straightforward. We propose storyboards, enriched by metadata, to specify functional and nonfunctional requirements. Accompanying tool support should facilitate the creation and use of storyboards. We used a meta-storyboard for the verification of storyboarding approaches.
\end{abstract}

\section{Introduction}

We expect the quality of a software product to improve on various aspects when people with complementary know-how are involved in user-centred software engineering (UCSE) approaches (e.g. software engineers, graphic designers, usability engineers, stakeholders, end users). However, ambiguity of (both functional and non-functional) requirements, because of different disciplines involved in the early stages of the development process, leads to undesired behavior of the final application [1].

An earlier literature study of user-centred design (UCD) techniques and tools showed that few tools actually include support for the cooperation of several disciplines [2]. It also revealed a lack of notations and tools for early stages of UCSE.

In order to verify whether our findings correspond with common practice, we interviewed companies that are active in UCD. These companies have between five and twenty years experience, employ five to forty professionals with various backgrounds, and accomplish projects in a wide range of application domains. Eight employees, mainly usability engineers and designers, participated in the interviews.

The interviewees often collaborate with external partners for the actual development of an application. With no exception, they indicated there is no sufficient support to translate their artefacts into a notation appropriate for software engineers or developers. Shortcomings of existing notations require close collaboration between all team members to avoid misconception.

Because of the independence of UCD relative to requirements engineering as known in software engineering [1], we investigate storyboarding for non-technical as 
well as technical team members. Two of the interviewed companies use storyboards, but only for specific applications, such as location-based systems or applications with a complex workflow. Nevertheless, they confirmed that storyboards are usable by all team members and contribute to later stages when suitable tool support should be available, which is confirmed in literature $[3,4]$. We propose a storyboarding tool for the creation and use of storyboards in multidisciplinary teams.

The storyboarding technique and our accompanying tool are described in the remainder of the paper. Furthermore, we propose the use of a meta-storyboard, depicting storyboarding in common practice, to evaluate and verify storyboarding in multidisciplinary teams.

\section{Storyboards as a Common Language in UCSE}

A suitable notation to support the early stages of UCSE needs to be (1) understandable for all team members, and have the possibility to (2) present both functional and non-functional requirements. Recent studies [3] show that the combination of stories and sketches is helpful to reason about a future application, errors, temporal information and contextual information. Consequently, scenarios, which are frequently used in UCSE and describe the future use of a system in narrative stories, become a much more powerful tool if visual information is added.

A storyboard consists of scenes that are related to each other and visualise sequences in a narrative scenario of use. This visualisation makes the narrative story more explicit and invokes empathy by depicting end users that interact with the future system [4]. Starting from a storyboard composed of scenes, we propose that each team member can add metadata according to their expertise. Metadata is similar to the free annotations designers usually add to storyboards [5], and concerns persona information resulting from a user needs analysis, device specifications or other annotations.

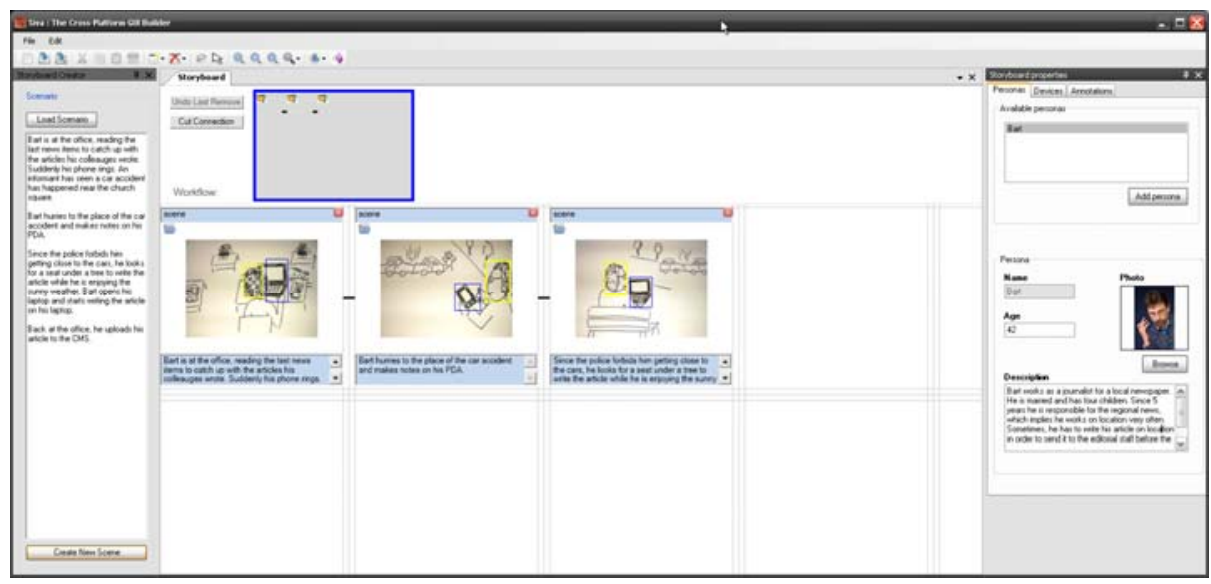

Fig. 1. A screenshot of our storyboarding tool, showing the narrative scenario (left panel), the storyboard with highlighted metadata (center) and the metadata details (right panel) 
The combination of storyboards and metadata can contribute to later stages of the UCSE process: device information provides input and assistance for the UI design; persona information supports roles in the UI design and the evaluation of designs. As such, the storyboard is used as a common language for the requirements elaboration phase. Integrated in our research tool, that supports several types of artefacts that are created within a multidisciplinary team, storyboards enriched by metadata can increase the visibility and traceability of a UCSE project. Furthermore, understandability is maintained for all team members.

Our storyboarding tool (Fig. 1) features the possibility to load a narrative scenario. Storyboards can be composed by adding scenes that are aligned with a particular sequence of the scenario. For each scene, a sketch or photo depicting end users interacting with the future application can be loaded. Metadata can be highlighted in each scene, while the metadata information can be entered in a form that is related to this highlighted part of the scene. Storyboards enriched by metadata avoid ambiguities in the scenario and provide connections to later stages such as the UI design.

\section{Storyboard Usage Exploration}

To verify if the proposed storyboarding approaches fit in common practice, we created a storyboard describing the usage of storyboards in a multi-disciplinary team. This meta-storyboard is based on the aforementioned interviews with practitioners and describes how storyboards are used in all stages of UCSE. The accompanying personas personify the basic disciplines involved in UCSE projects.

In order to verify the concept of storyboarding in an entire UCSE process, we evaluated the meta-storyboard in an informal test consisting of two phases: an evaluation of the storyboarding tool and a walkthrough to validate the meta-storyboard. The latter additionally enabled us to verify the suitability of storyboards and the tool to discuss the use of a future application. Four female and three male subjects participated. Their backgrounds were very diverse, including interaction design, graphic design, computer science and media studies. The subjects' experience in multidisciplinary project teams ranged from a few months to more than five years.

After the subjects got a first impression of storyboarding and the transition to UI design using the tool, they participated in the meta-storyboard walkthrough. Each subject was asked to keep in mind a given persona description while discussing the meta-storyboard scene by scene using the tool. The discussions were recorded using a voice recorder and after the walkthrough, the subjects answered a questionnaire concerning the persona description and the use of storyboards.

The visual representation of the meta-storyboard stimulated discussion and the tool facilitated the understanding: clicking the metadata available in a scene enabled the exploration of personas and devices. After the walkthrough, all subjects declared it was easy to understand the approach depicted in the meta-storyboard and they all confirmed that the persona information contributed to this understanding.

All subjects agreed with the general approach presented by the storyboard. They accepted the use of storyboards in a multidisciplinary team. Most subjects prefer various disciplines to be involved in the creation of the storyboard to avoid misunderstandings at later stages. The technical subjects confirmed their work would benefit 
from storyboards because storyboards and provide more explicit information concerning activities carried out by users. Four subjects noticed that the tool should adapt its view and metadata according to the background of the team member working with the tool or the purpose of the storyboard at a particular stage.

\section{Conclusion}

The survey, we held among companies involved in UCD, inspired us to introduce storyboarding in multidisciplinary teams to specify functional and non-functional requirements. Storyboarding and tool support for storyboarding, were evaluated using a meta-storyboard that visualises the use of storyboards in a multidisciplinary team.

Our meta-storyboard was a valuable artefact for discussion and proved to be very understandable for team members with different backgrounds. The metadata, highlighted in the tool, contributed to the understanding of the meta-storyboard. The metastoryboard walkthrough revealed that the tool should provide various visualizations according to the team members' backgrounds and the purpose of the storyboard. These and other results will be incorporated in the storyboarding tool. In the future we will frequently consult practitioners to investigate how metadata of storyboards contributes to UCSE projects and to adapt the storyboarding tool to common practice.

Acknowledgments. Part of the research at EDM is funded by the ERDF (European Regional Development Fund) and the Flemish Government. The research described in this paper is based on our experiences in IWT project AMASS++ (IWT 060051).

\section{References}

[1] Lindgaard, G., Dillon, R., Trbovich, P., White, R., Fernandes, G., Lundahl, S., Pinnamaneni, A.: User needs analysis and requirements engineering: Theory and practice. Interact. Comput. 18(1), 47-70 (2006)

[2] Haesen, M., Coninx, K., Van den Bergh, J., Luyten, K.: MuiCSer: A Process Framework for Multi-Disciplinary User-Centered Software Engineering processes. In: Proc. of Human-Centred Software Engineering, September 2008, pp. 150-165 (2008)

[3] Brown, J., Lindgaard, G., Biddle, R.: Stories, Sketches, and Lists: Developers and Interaction Designers Interacting Through Artefacts. In: Proc. of Agile 2008, pp. 39-50 (2008)

[4] Truong, K.N., Hayes, G.R., Abowd, G.D.: Storyboarding: an empirical determination of best practices and effective guidelines. In: DIS 2006: Proc. of the 6th conference on Designing Interactive systems, pp. 12-21. ACM Press, New York (2006)

[5] Myers, B.A., Park, S.Y., Nakano, Y., Mueller, G., Ko, A.: How designers design and program interactive behaviors. In: VL/HCC, pp. 177-184 (2008) 